\title{
SUMO-triggered ubiquitination of NR4A1 controls macrophage cell death
}

\author{
Long Zhang ${ }^{*, 1,4}$, Feng Xie ${ }^{2,4,}$, Juan Zhang ${ }^{3}$, Peter ten Dijke ${ }^{3}$ and Fangfang Zhou ${ }^{\star, 2}$
}

Nuclear receptor NR4A1 has been implicated as a key regulator in a wide range of pathophysiological responses. As an immediate early response gene, NR4A1 can be rapidly and potently induced by a variety of stimuli. Its induction is followed by its rapid degradation, but the mechanism by which NR4A1 is degraded remains poorly understood. Here we show that nuclear receptor NR4A1 is sumoylated by SUMO2/3. Upon poly-SUMO modification, NR4A1 can be targeted by the SUMO-dependent E3 ubiquitin ligase RNF4 for polyubiquitination and subsequent degradation. The SUMO E3 ligase PIAS3 promotes SUMOylation and polyubiquitination of NR4A1, while the SUMO protease SENP1 acts to de-conjugate SUMO. We demonstrate that this pathway is important for rapid degradation of NR4A1 after induced by stress. Moreover, we identify two SUMO modification sites in NR4A1 that are critical for maintaining low levels of NR4A1 expression. Mutation of these two NR4A1 SUMO modification sites enhances the stability of NR4A1. Importantly, we show that SUMOylation is critical in controlling NR4A1 function in inflammatory cytokine signaling and controlling macrophage cell death. SUMOylation and subsequent ubiquitination on NR4A1 mitigates its inhibition of innate immune signaling, such as TNF- $\alpha$ - and IL- $1 \beta$-induced NF- $\kappa$ B activation. This mechanism of sequential SUMOylation and ubiquitination, which together control the degradation of NR4A1, could be exploited for the therapeutic treatment of diseases with NR4A1 involvement.

Cell Death and Differentiation (2017) 24, 1530-1539; doi:10.1038/cdd.2017.29; published online 16 June 2017

SUMO modification, which involves the covalent attachment of SUMO to a diverse array of proteins, regulates a wide range of cellular processes, including transcription, replication, chromosome segregation, and DNA repair. SUMO, which stands for small ubiquitin-related modifier, becomes conjugated to its substrates via the heterodimeric SUMO E1 activating enzyme, a single SUMO E2 (UBE2I) conjugating enzyme, and a restricted set of E3 protein ligases that include 'protein inhibitor of activated STAT' (PIAS) family members. ${ }^{1,2}$ Although the E1 and E2 enzymes alone can modify most substrates in vitro, an E3 ligase is usually required for selective and effective recognition of substrates in vivo. ${ }^{3-5}$ SUMO-specific proteases (SENPS) can cleave SUMO from target proteins, making SUMOylation a reversible and dynamic process. ${ }^{6}$ In most cases, SUMO modification occurs within a consensus modification site $(\psi \mathrm{KxD} / \mathrm{E}$, where $\psi$ represents a bulky hydrophobic group). ${ }^{7}$ Similar to ubiquitin, SUMO can be covalently linked and thus form chains (polySUMO) via internal lysine SUMOylation sites. , $^{8}$

Four SUMO isoforms, termed SUMO1-4, have been identified in vertebrates. SUMO1, -2 , and -3 are conjugated to distinct substrates in vivo, and SUMO2 and -3 are very similar in terms of structure and function. At present, it remains unclear whether SUMO4 is conjugated to cellular proteins. SUMO2 and -3 have a lysine at position 11 in a consensus sequence. ${ }^{10}$ SUMO1 lacks such a site and does not contribute efficiently to SUMO chains. Proteins that are SUMOylated can associate in a non-covalent manner with other proteins via their SUMO interaction motifs (SIMs). Unlike lysine 48-mediated ubiquitination, SUMOylation does not directly target its substrates to proteosomal degradation. However, recent studies demonstrated that poly-SUMO-modified proteins can trigger degradation through the ubiquitin-mediated proteasome pathway and indirectly through SUMOtargeted SIM (SUMO-interacting motif) domain-containing ubiquitin ligases, such as the RING finger protein RNF4 and RNF111, which contains several SIMs. ${ }^{8,11-16}$

Nuclear receptor 4A (NR4A1), which is also called Nur77, TR3, and NGF-IB, is a member of the steroid nuclear hormone receptor superfamily. It is part of the NR4A group, which includes NR4A2 and NR4A3. In contrast to classic steroid receptors that require ligand activation, NR4A family members are constitutively active. ${ }^{17,18} \mathrm{NR} 4 \mathrm{~A}$ plays a critical role in a large spectrum of pathophysiological processes. ${ }^{19-22}$ Specifically, NR4A is an immediate early response gene that can be induced in different cell types and tissues by growth factors, stimuli, and stress. ${ }^{23-25}$ NR4A1 and NR4A3 are required for T-cell receptor-mediated apoptosis. ${ }^{26}$ Although NR4A1 and NR4A3 single-knockout mice do not exhibit any thymic or peripheral T-cell death, NR4A1 and NR4A3 double-knockout mice develop acute myeloid leukemia, revealing the redundant functions of these family members. ${ }^{27}$ Reduced NR4A gene dosage leads to mixed myelodysplastic/myeloproliferative neoplasms in mice. ${ }^{28} \mathrm{NR} 4 \mathrm{~A} 1$ is also involved in the regulation

\footnotetext{
${ }^{1}$ Life Sciences Institute, Zhejiang University, Hangzhou, Zhejiang 310058, China; ${ }^{2}$ Institutes of Biology and Medical Science, Soochow University, Suzhou 215123 , PR China and ${ }^{3}$ Department of Molecular Cell Biology and Centre for Biomedical Genetics, Leiden University Medical Center, Postbus 96002300 RC Leiden, The Netherlands ${ }^{*}$ Corresponding author: F Zhou, Institutes of Biology and Medical Science, Soochow University, Suzhou 215123, PR China. Tel: +86 512 65882491 ; E-mail: zhoufangfang@suda.edu.cn

or L Zhang, Life Sciences Institute and Innovation Center for Cell Signaling Network, Hangzhou, Zhejiang 310058, China. E-mail: I.zhang@lumc.nl

${ }^{4}$ These authors contributed equally to this work.

Received 15.9.16; revised 02.2.17; accepted 17.2.17; Edited by C Borner; published online 16.6.17
} 
of apoptosis and cell death in several cell types. ${ }^{29,30}$ Specifically, apoptotic stimuli cause the translocation of NR4A1 from the nucleus to the mitochondria, which is followed by the induction of cytochrome c release. ${ }^{31,32}$ In addition, NR4A1 prevents atherogenesis and regulates hepatic glucose metabolism in a mouse model. ${ }^{33-35}$

Because NR4A1 has multiple functions, its stability and activity is likely to be carefully regulated. Several studies have shown that NR4A1 transcriptional activity and stability can be regulated by a series of coactivators SRC-2, PCAF, p300, DRIP250, TRAP220 and Pin1, as well as by the corepressors SHP and CRIF1 (refs 31,32,36-38). The post-translational modifications, like phosphorylation and acetylation have also been demonstrated to be important for the activity and function of NR4As. ${ }^{37,39-41}$ Stimuli such as epidermal growth factor, nerve growth factor, and phorbol myristate acetate can induce NR4A1 phosphorylation, ${ }^{42,43}$ and this phosphorylation is mediated by kinases such as Akt, pp90RSK, and Chk2 (refs $39,40,44)$.

Notably, the mechanism by which NR4A1 is rapidly degraded after induction is not well understood. Here we show that the nuclear receptor NR4A1 undergoes SUMOtriggered ubiquitin-mediated degradation that is dependent on the SUMO-targeted ubiquitin ligase RNF4. Further, we show that SUMOylation is critical in controlling NR4A1 protein stability and function in inflammatory cytokine signaling.

\section{Results}

NR4A1 undergoes SUMO modification. To investigate the regulation of $\mathrm{NR} 4 \mathrm{~A} 1$, we examined its possible posttranslational modification by SUMO1, -2 and -3 in HEK293T cells transfected with NR4A1 and SUMO1-3. As shown in Figure 1a, ectopically expressed NR4A1 underwent SUMO2/3 SUMOylation at a very low level. Strikingly, after treatment with a proteasome inhibitor, NR4A1-SUMO2/3 conjugation increased dramatically. NR4A1-SUMO1 was not detectable (Figure 1a). Importantly, analysis of non-transfected cells demonstrated that NR4A1 was also conjugated by SUMO2/3 in non-transfected cells (Figure 1b). Taken together, these data indicate that NR4A1 undergoes SUMO2/3 but not SUMO1-mediated SUMOylation and that this post-translational modification by SUMO is coupled to proteasome-mediated degradation of NR4A1.

NR4A1 interacts with the SUMO E3 ligase PIAS3. PIAS is specific SUMO E3 ligase. When we performed a co-immunoprecipitation in HEK293T cells transfected with NR4A1 and a panel of SUMO E3 ligases, the PIAS3 ligase showed the strongest interaction with NR4A1, while PIAS1 and PIASXa interacted only weakly with NR4A1 (Figure 2a). These results were further validated by half endogenous immunoprecipitation showing that Flag-PIAS3 co-immunoprecipitated with endogenous NR4A1 and that endogenous PIAS3 interacted with endogenous NR4A1 (Figures $2 b$ and $\mathrm{c}$ ). Consistent with this, we found that the two proteins co-localized in the nucleus (Supplementary Figure S1). These data suggest that PIAS3 may function as a SUMO E3 ligase for NR4A1.

SUMO modification of NR4A1 triggers polyubiquitination and is stimulated by PIAS3 and reversed by SENP1. Consistent with the notion that PIAS3 is a SUMO E3 ligase, we observed that forced SUMO3 expression increased the SUMO modification of NR4A1 (Figure 3a). Next, we examined the interactions between NR4A1 ubiquitination and SUMOylation. Increased SUMO3-modification of NR4A1 led to a concomitant increase in NR4A1 polyubiquitination (Figure 3a). In addition, the PIAS3-induced poly-SUMO chain conjugation to NR4A1 coincided with increased poly-ubiquitin chain modification. The PIAS3-mediated induction of ubiquitin/SUMO chain modification of NR4A1 led to a decrease in a

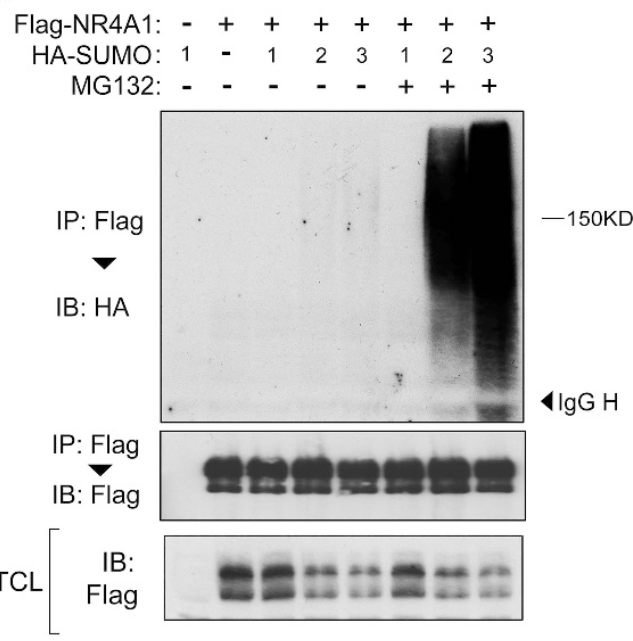

b

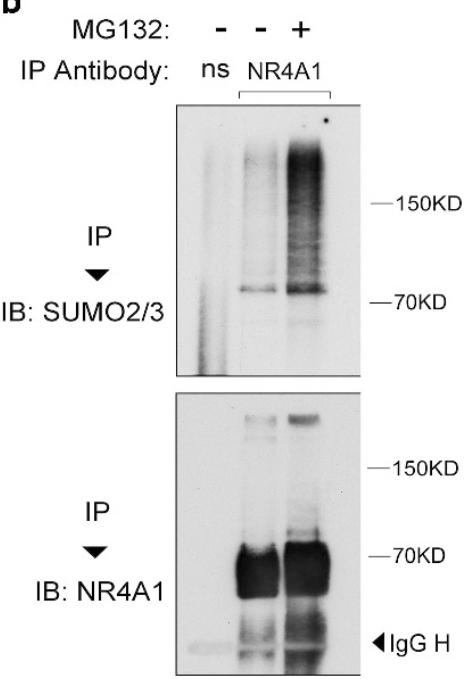

Figure 1 NR4A1 undergoes SUMO2/3-mediated SUMOylation. (a) Immunoblot (IB) analysis of immunoprecipitated (IP) cell lysate from HEK293T cells that were transfected with Flag-NR4A1 plus HA-SUMO1, -2 or -3 and either left untreated or treated for $5 \mathrm{~h}$ with $5 \mu \mathrm{M}$ MG132. (b) Untreated or MG132-treated HeLa cells were collected, subjected to IP using anti-NR4A1 antibody, and analyzed by SUMO2/3 IB 


$$
\text { a }
$$

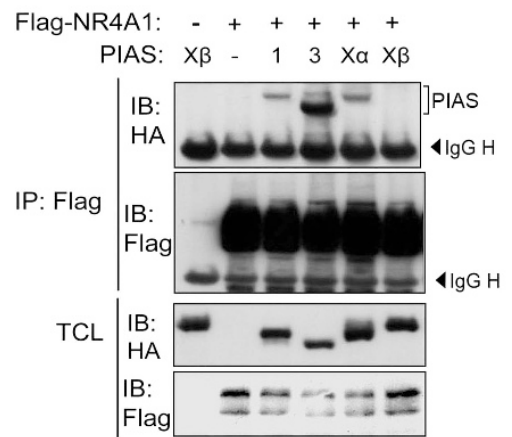

b

IB: NR4A1
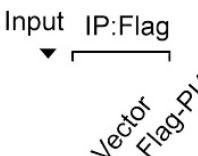

$p^{s^{3}}$

IP Antibody

IB:Flag

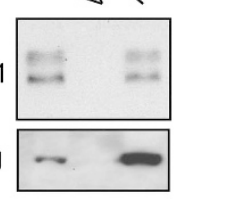

IB: PIAS3

IB: NR4A1

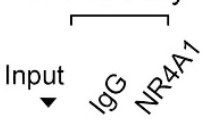

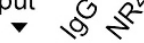

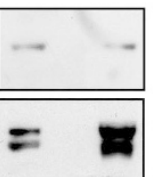

Figure 2 NR4A1 interacts with the SUMO E3 ligase PIAS3. (a) HEK293T cells were transfected with Flag-NR4A1 and HA-PIAS as indicated. Cells were collected for immunoprecipitation (IP) with Flag M2 beads and subjected to immunoblot (IB) analysis. (b) IP and IB analysis of HEK293T cells transfected with control vector or with Flag-PIAS3. (c) HEK293T cells were collected for IP with NR4A1 antibody followed by PIAS3 IB analysis

a

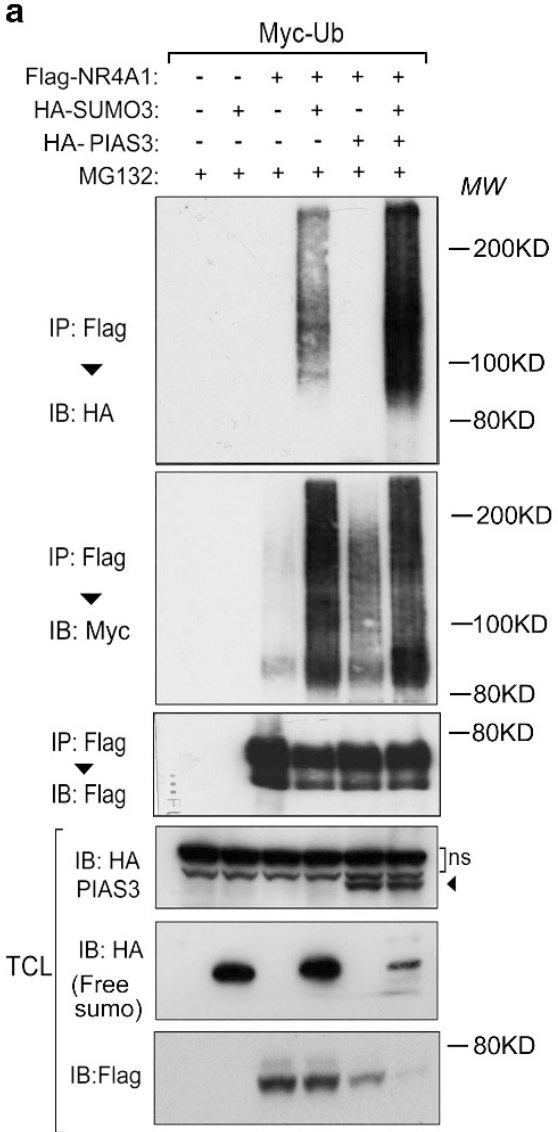

b

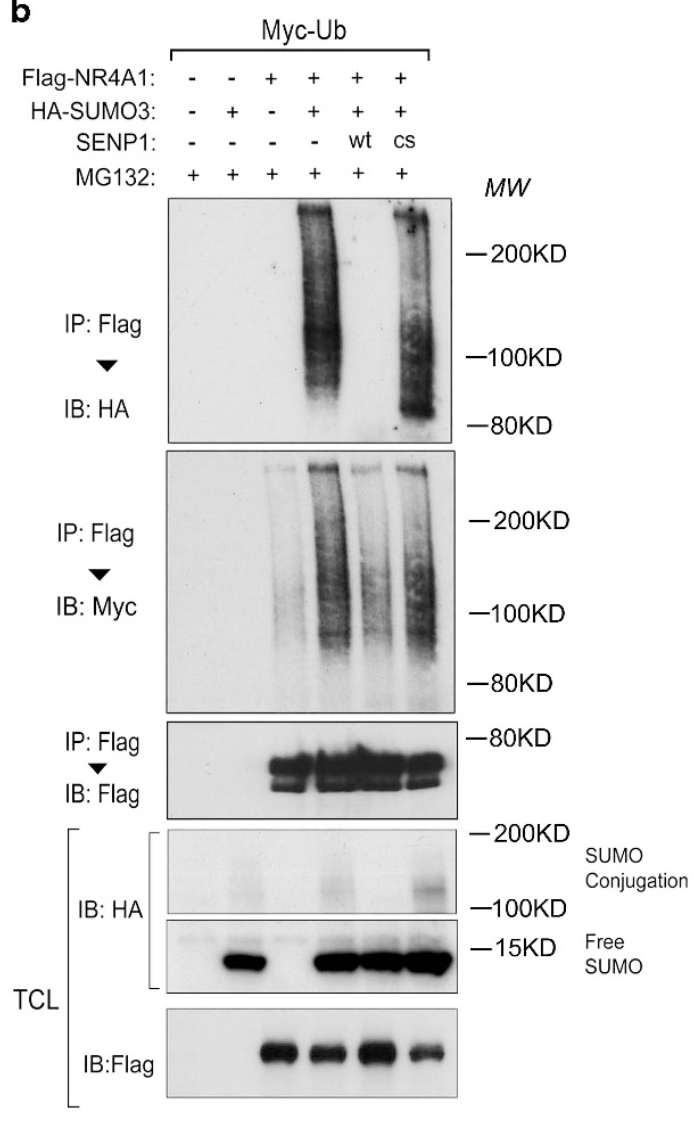

Figure 3 The SUMO E3 ligase enhances NR4A1 SUMOylation, and the deSUMOylation enzyme decreases NR4A1 SUMOylation. (a and b) HEK293T cells stably expressing Myc-Ub were transfected with Flag-NR4A1, HA-SUMO3, and PIAS3 (a) or with SENP WT/CS (b) as indicated. Cells were treated with the proteasome inhibitor MG132 for ( $5 \mu \mathrm{M}$ for $5 \mathrm{~h}$ ) and then collected for immunoprecipitation (IP) and immunoblot (IB) analysis

the levels of non-SUMO- or ubiquitin-modified NR4A1 (Figure 3a).

SUMOylation is a reversible process, and SENPs have been implicated as deconjugating enzymes in this process. We investigated whether SUMO modification and SUMO modification-triggered polyubiquitination of NR4A1 could be reversed by SENPs. Wild-type SENP1 completely blocked
NR4A1-SUMO3 conjugation (Figure 3b). This effect was critically dependent on SNEP1 protease activity, as shown by the observation that the SENP1 CS mutant, which lacks the deconjugation enzymatic activity, did not have the same effect as wild-type SENP1. In addition, SUMO conjugation-triggered polyubiquitination of NR4A1 was clearly decreased by wildtype SENP1 but not by the SENP1 CS mutant (Figure 3b). 


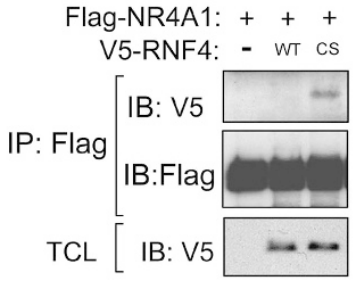

d

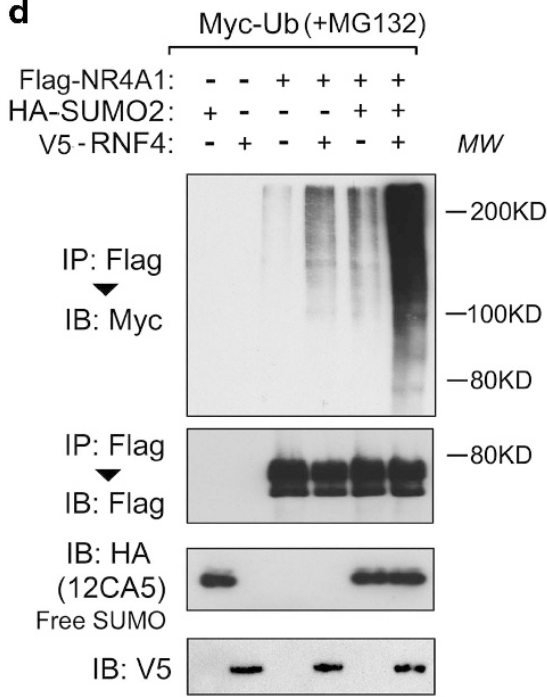

b

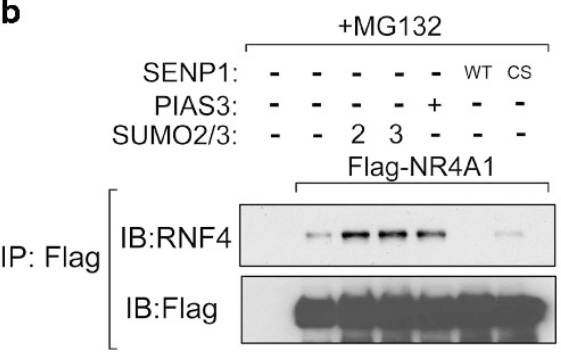

e

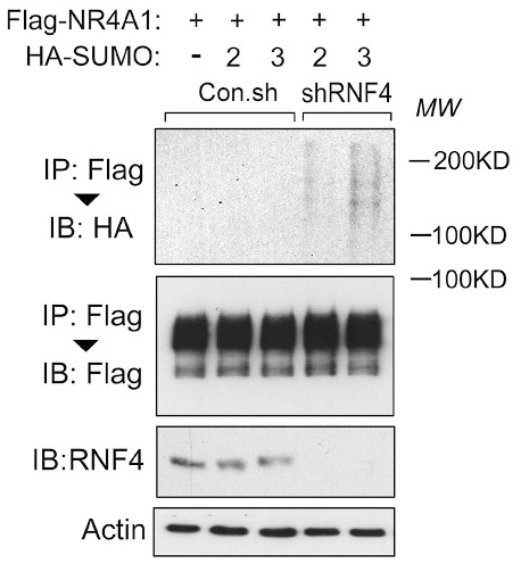

c

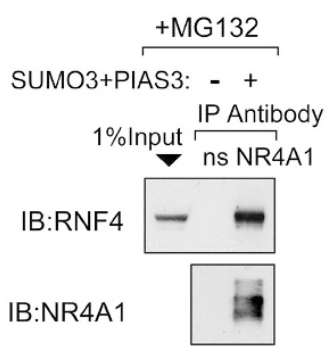

$\mathbf{f}$

Flag-NR4A1: $\quad-\quad++++$

HA-SUMO2: +--++

shRNF4: $\quad-+-++M W$

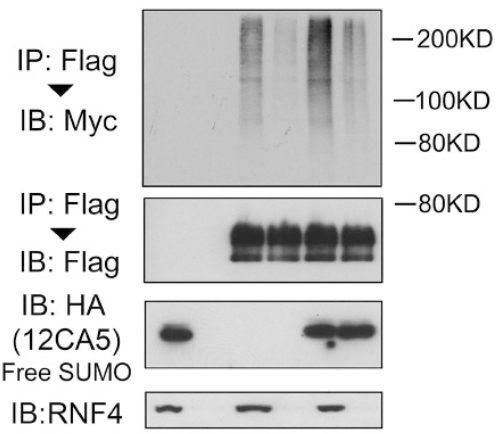

Figure 4 RNF4 is required for SUMOylation-triggered polyubiquitination of NR4A1. (a) HEK293T cells transfected with Flag-NR4A1 or V5-RNF4 WT/CS as indicated were collected for immunoprecipitation (IP) and immunoblot (IB) analysis. (b) HEK293T cells were transfected with or without SENP1 WT/CS, PIAS3, or SUMO2/3 as indicated. Cells were treated with the proteasome inhibitor MG132 $(5 \mu \mathrm{M}$ for $5 \mathrm{~h})$ and then collected for IP and IB analysis. (c) Endogenous interactions between NR4A1 and RNF4 in HEK293T cells transfected with SUMO3 and PIAS3. Cells were treated with MG132 ( $5 \mu \mathrm{M}$ for $5 \mathrm{~h}$ ) before harvesting. (d) HEK293T cells expressing Myc-Ub were transfected with Flag-NR4A1, HA-SUMO2, or RNF4 as indicated. Cells were treated with MG132 ( $5 \mu \mathrm{M}$ for $5 \mathrm{~h}$ ) and collected for IP and IB analysis. (e) RNF4 depletion leads to the accumulation of NR4A1 SUMO2/3 conjugates in the absence of MG132 treatment in HEK293T cells. shRNF4, small hairpin RNA-targeting RNF4. (f) HEK293T cells expressing Myc-Ub were transfected with Flag-NR4A1 and HA-SUMO and infected with the RNF4 shRNA lentivirus. Cells were treated with MG132 $(5 \mu \mathrm{M}$ for $5 \mathrm{~h})$ and collected for IP and IB analysis

Taken together, these data indicate that SUMO modification of NR4A1 triggers its polyubiquitination and that this process is reversible. Thus, the extent of NR4A1 SUMO modification seems to be balanced by the activities of the SUMO-E3 ligase PIAS3 and the SUMO deconjugating enzyme SENP1.

RNF4 mediates SUMO modification-triggered polyubiquitination of NR4A1. We next tried to identify the E3 ubiquitin ligase that mediated the SUMO-triggered polyubiquitination of NR4A1. Several Ring finger proteins have been reported to have a SIM, ${ }^{45,46}$ so we focused on these particular E3 ligases as a possible candidate for interacting with SUMOylated NR4A1. In the absence of the proteasome inhibitor MG132, NR4A1 interacted weakly with the E3 ligase RNF4 CS mutant (which lacks E3 ligase activity) but not with wild-type RNF4 (Figure 4a). SUMO chain-induced dimerization activates RNF4 (ref. 47). Thus, RNF4 might interact transiently with SUMOylated-NR4A1 and mediate its degradation. To test this, we investigated the interaction between NR4A1 and RNF4 in the presence of MG132. Ectopically expressed NR4A1 bound endogenous RNF4, and this interaction was strengthened by co-expression with SUMO2/3 or with the
SUMO-E3 ligase PIAS3 (Figure 4b), both of which stimulate the SUMOylation of NR4A1. Importantly, the NR4A1-RNF4 interaction was disrupted in cells that were transfected with wild-type SUMO-deconjugating enzyme SENP1 but not in cells transfected with SENP1 CS (Figure 4b). Endogenous interactions between NR4A1 and RNF4 could only be detected in cells that were co-transfected with SUMO3 and PIAS3 (Figure 4c). These results suggest that RNF4 recognizes SUMO-conjugated NR4A1.

We next performed an ubiquitination assay to verify the involvement of RNF4 in the SUMO-triggered ubiquitination of NR4A1. Ectopic expression of RNF4 promoted polyubiquitination of NR4A1, and this effect was clearer when exogenous SUMO was co-expressed with RNF4 (Figure 4d). In the absence of MG132 treatment, RNF4 depletion caused the accumulation of NR4A1-SUMO conjugates that had higher molecular weights (Figure 4e). Furthermore, RNF4 depletion reduced basal and exogenous SUMO-triggered NR4A1 ubiquitination (Figure 4f). These results suggest that the SUMOylation-triggered polyubiquitination of NR4A1 was mitigated by RNF4 depletion. Taken together, these data 


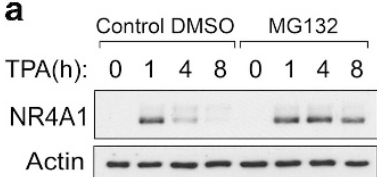

b

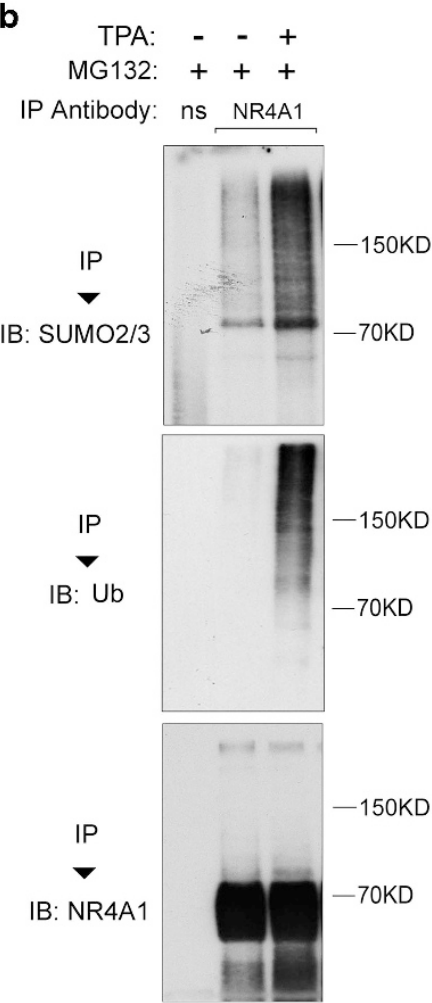

C

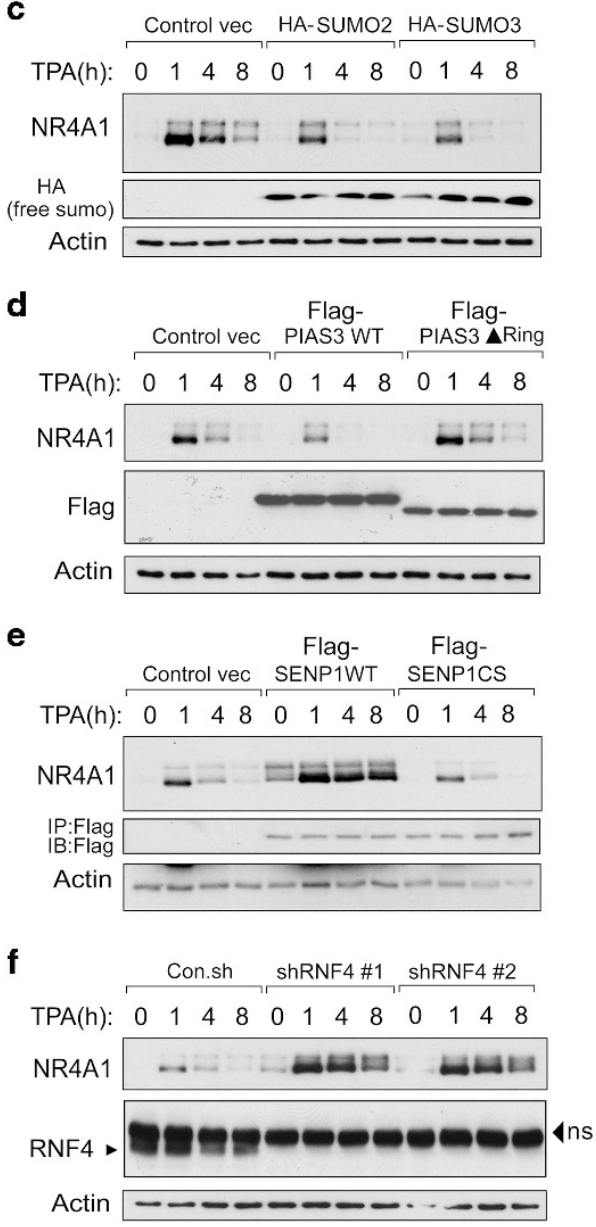

Figure 5 NR4A1 expressed in response to TPA stimulation undergoes SUMOylation-mediated degradation. (a) HEK293T cells were treated with DMSO or the proteasome inhibitor MG132 (5 $\mu \mathrm{M}$ for $5 \mathrm{~h}$ ) along with TPA (100 ng/ml) for the indicated time. Cells were collected for immunoblot (IB) analysis. (b) HEK293T cells were treated with TPA $(100 \mathrm{ng} / \mathrm{ml})$ and MG132 $(5 \mu \mathrm{M})$ as indicated for $5 \mathrm{~h}$. Cells were collected for immunoprecipitation (IP) with non-specific (ns) or anti-NR4A1 antibody followed by IB analysis. (c) HEK293T cells were transfected with control vector, SUMO2, or SUMO3 plasmids as indicated. Cells were treated with TPA for the indicated time and collected for IB analysis. (d) PIAS3 WT but not the PIAS3 $\triangle$ Ring mutant accelerated the degradation of the induced NR4A1 in HEK293T cells. (e) IB analysis of cell lysate from HEK293T cells transfected with control vector, SENP1 WT, or the SENP1 CS mutant and treated with TPA (100 ng/ml) for the indicated time. (f) RNF4 depletion using two independent shRNAs (\#1 and \#2) increased and extended TPA-induced NR4A1 expression in Jurkat cells. shRNF4, small hairpin RNA-targeting RNF4. NS, non-specific. Actin was included as a loading control in (a) and in (c-f)

show that RNF4 mediates the polyubiquitination of SUMO-modified NR4A1.

\section{Stress-induced NR4A1 degrades quickly via SUMOylation-triggered polyubiquitination and subse- quent degradation. Having demonstrated that NR4A1 undergoes SUMOylation-triggered polyubiquitination, we next wanted to determine the physiological significance of this finding. Basal NR4A1 expression is low in vivo, but expression is induced rapidly in different cell types and organs by many types of stimuli, including serum, inflamma- tory factors, growth factors, phorbol ester, and stress ${ }^{48}$. We observed that NR4A1 expression in HeLa and HEK293T cells is rapidly induced in response to 12-O-Tetradecanoylphorbol- 13-acetate (TPA) and is rapidly degraded afterwards. How- ever, when cells were challenged with the proteasome inhibitor MG132, the TPA-induced NR4A1 expression was sustained (Figure 5a and data not shown). These results}

confirm previous reports and suggest that stress-induced NR4A1 proteins are rapidly targeted for degradation via the proteasome. TPA treatment enhanced PIAS3-NR4A1 interaction and greatly increased SUMO modification and the polyubiquitination of NR4A1 (Figure 5b and Supplementary Figure S2). Thus, SUMOylation-triggered polyubiquitination plays an important role in depleting stress-induced NR4A1 protein from cells.

To confirm this result, we expressed or knocked down the expression of proteins that are responsible for SUMO modification or SUMO modification-trigged polyubiquitination in cells. TPA-induced NR4A1 degraded faster in SUMO2/3transfected cells than in control cells (Figure $5 \mathrm{c}$ ). SUMO E3 ligase PIAS3 (PIAS3 WT), but not ring domain-deleted mutant of PIAS3 (PIAS3 $\triangle$ Ring), also accelerated NR4A1 degradation (Figure 5d). Consistent with these findings, ectopic SENP1 (SENP1 WT) expression strongly and stably increased both basal and TPA-induced NR4A1 expression, 
while the SENP CS mutant did not have this effect (Figure 5e). RNF4 depletion by two independent shRNAs exhibited effects similar to those of wild-type SENP1 (Figure 5f). Given a pivotal role of the NR4A receptors in regulating immune cell phenotype, we examined whether this new regulatory system also occurs in human monocytes. As shown in THP-1 cells, LPS stimulation rapidly induced NR4A1 expression and the induced NR4A1 level was sustained by MG132 treatment (Supplementary Figure S3a). Similarly, the LPS-induced NR4A1 also degraded faster in cells expressing PIAS3 WT, but not in cells expressing PIAS3 $\triangle$ Ring mutant (Supplementary Figure S3b). Consistently, LPS-induced NR4A1 was stabilized by ectopic expression of SENP1 WT (but not the CS mutant) and depletion of either PIAS3 or RNF4 (Supplementary Figure S3c-e).

In line with this, PIAS3 inhibited and SENP1 enhanced the transcriptional activity of NR4A1, while their mutants PIAS3 ARing and SENP1 CS lost these effects. RNF4 overexpression and knockdown repressed and upregulated NR4A1 transcriptional activity, respectively (Supplementary Figure S4). Taken together, our data demonstrate that the degradation of stimuli-induced NR4A1 can be accelerated by SUMO modification-triggered polyubiquitination and can be inhibited by reversing this process.

Mutating two consensus SUMO modification sites stabilizes NR4A1. In most cases, SUMO modification occurs within a consensus modification site $(\psi \mathrm{KxD} / \mathrm{E}$, where $\psi$ represents a bulky hydrophobic group). We analyzed the protein sequence of NR4A1 and found two such consensus sites (Figure 6a). Although single mutation of either site (K101R or K577R) did not change the NR4A1 SUMO modification or polyubiquitination, these modifications of NR4A1 were absent when both sites were mutated (K101R and K577R) (Figure 6b). Mutation of both sites in NR4A1 also abrogated the interaction of NR4A1 with RNF4 (Figure 6c). Thus, NR4A1 proteins that lack SUMO modification did not associate with RNF4 and could not be targeted for polyubiquitination by RNF4.

After mutating $\mathrm{K}$ to $\mathrm{R}$ at these two sites, the half-life of NR4A1 was extended substantially (Figure 7a). In line with this, mutant NR4A1 (K101/577R) had higher transcriptional activity than wild-type NR4A1 (Figure 7b). NR4A1 negatively regulates TNF- $\alpha$ - and IL- $1 \beta$-induced NF- $k B$ activation by transcriptionally targeting $\mathrm{I}_{\kappa} \mathrm{B} a$ expression. ${ }^{34}$ Compared to wild-type NR4A1, the K101/577R mutant induced higher expression of $\mathrm{I}_{K} \mathrm{Ba}$ (Figure $7 \mathrm{c}$ ) and exhibited more potent repression of TNF- $\alpha-$ and IL- $1 \beta$-induced NF- $K B$ activity (Figure $7 d$ ). NF- $k B$ activation is involved in immune cell survival. ${ }^{49,50}$ Consistent with this, the NR4A1 K101/577R mutant more efficiently induced the levels of active caspase 3 in human mynocyte THP-1 cells (Supplementary Figure S5). Annexin V/PI staining from flow cytometry (FACS) showed that ectopic expression of NR4A1 WT in THP-1 cells increased Annexin $\mathrm{V}$ positive cells and Annexin V/PI double positive cells; this effect was more obviously observed when THP-1 cells were ectopically expressed with NR4A1 K101/577R mutant (Figure 7e), suggesting that the SUMO modification-deficient NR4A1 K101/577R mutant could more potently induce apoptotic cell death in THP-1 cells. Taken together, these data show that lysine 101 and lysine 577 are the two major SUMO-conjugating sites in NR4A1; mutating both of these two sites stabilizes NR4A1 and increases its transcriptional regulation activity as well as its other biological activities.

\section{Discussion}

In this study, we elucidated the mechanisms by which the stability of the orphan nuclear receptor NR4A1 is regulated via SUMOylation and subsequent ubiquitination. SUMOylation of a

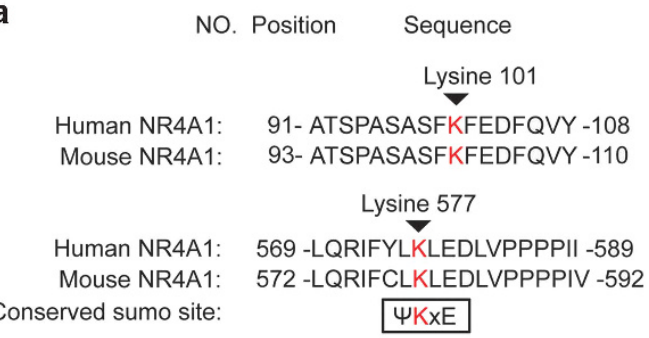

b

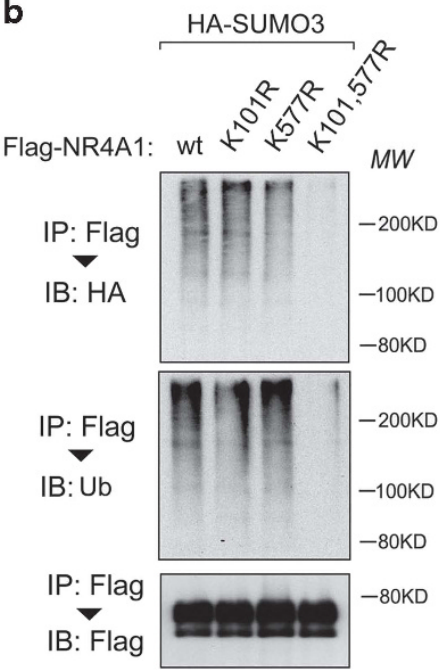

c

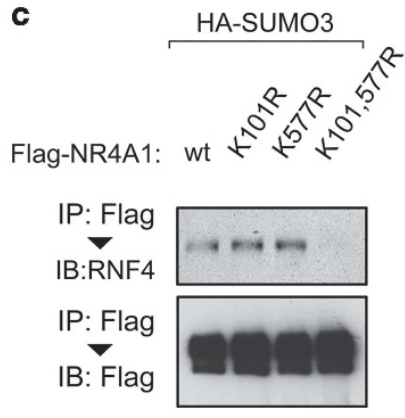

Figure 6 NR4A1 has two major SUMO modification sites. (a) Sequences and locations of putative NR4A1 SUMO modification sites in the human and mouse NR4A1 protein sequences. (b) Double mutation of the NR4A1 SUMO modification sites (K101/577 R) blocked both NR4A1 SUMOylation and polyubiquitination in HEK293T cells. (c) HEK293T cells expressing HA-SUMO3 were transfected with Flag-NR4A1 WT, single mutant (K101R or K577 R) and double mutant (K101/577R) as indicated and treated with the proteasome inhibitor MG132 (5 $\mu \mathrm{M}$ for $5 \mathrm{~h})$. Cells were then collected for immunoprecipitation (IP) and immunoblot (IB) analysis 
a
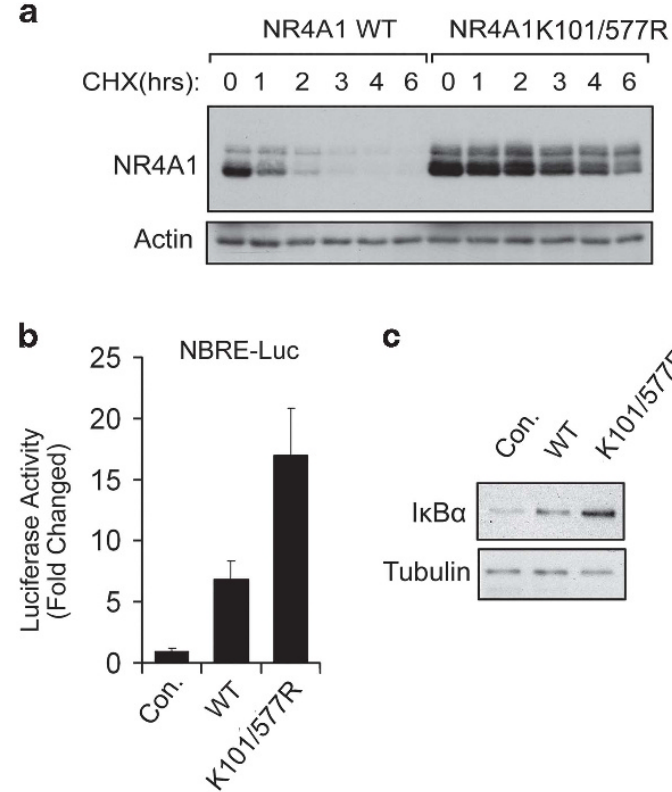

c

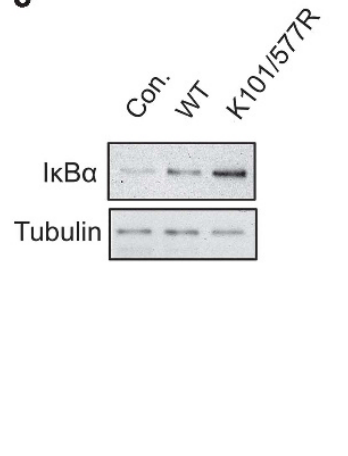

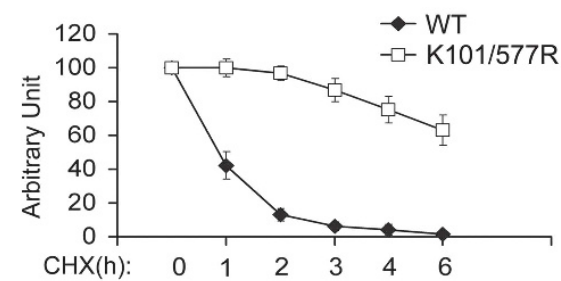

d

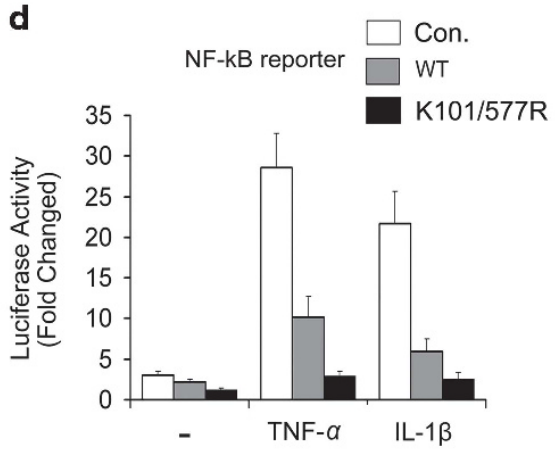

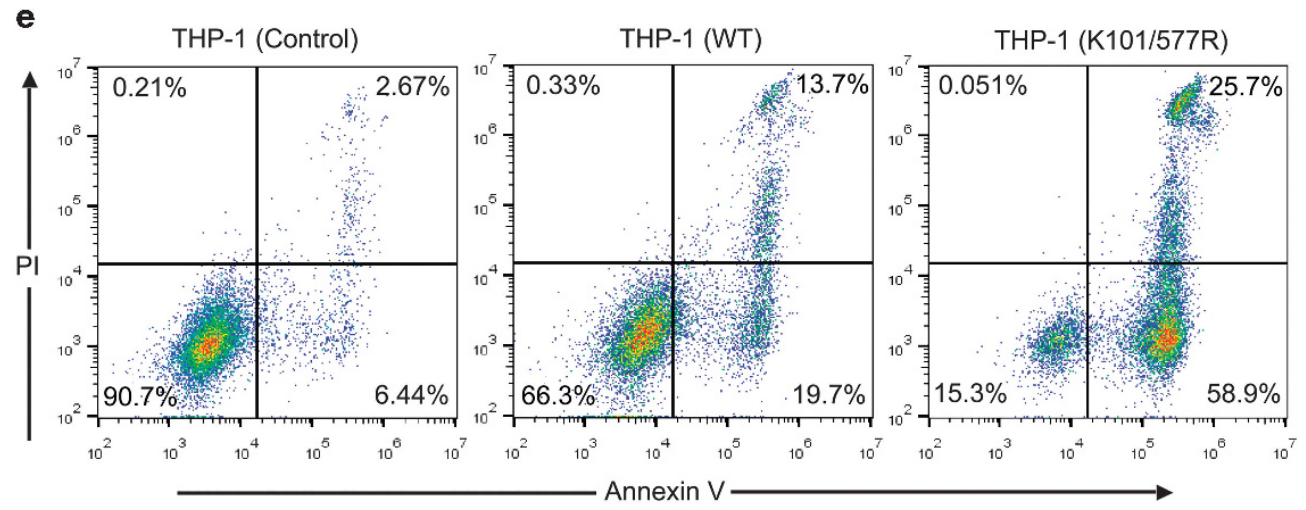

f

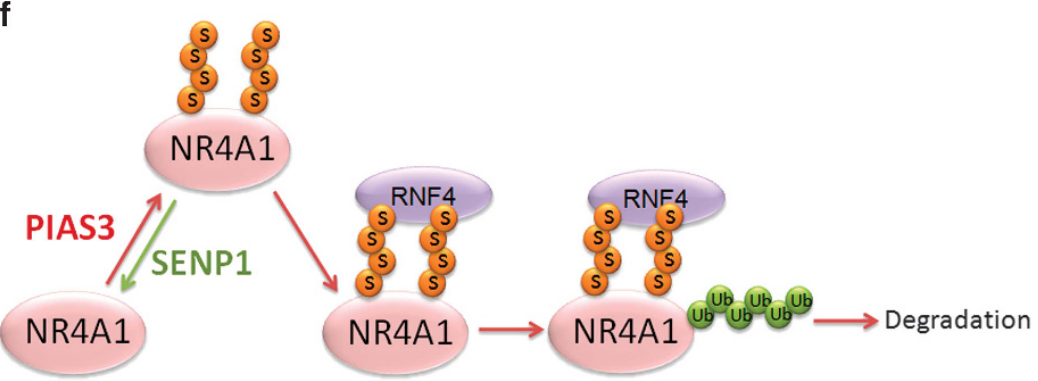

Figure 7 Mutation of two consensus SUMO modification sites stabilizes NR4A1 and increases its biological activities. (a) (Left panel) Immunoblot analysis shows that the NR4A1 K101/577 R double mutant extends the half-life of NR4A1 in HeLa cells. (Right panel) The degradation rate of NR4A1 WT and the NR4A1 double mutant K101/577 R are shown graphically after quantitative densitometry was performed. (b) Transcriptional activity analysis of wild-type (WT) NR4A1 and the double mutant K101/577 R using a NBRELuc reporter in HEK293T cells. (c) Immunoblot analysis of HeLa cells infected with lentivirus expressing control vector, NR4A1 WT, or the NR4A1 K101/577 R mutant. (d) NF- $\mathrm{KB}$ activity induced by TNF- $\alpha$ or IL-1 $\beta$ was inhibited more by the NR4A1 double mutant K101/577 R than by NR4A1 WT in HEK293T cells. (e) THP-1 cells infected with lentivirus expressing a control vector, NR4A1 WT or the K101, 577 R double mutant were stained with Annexin V and Propidium lodine (PI) and analyzed by FACS. (f) Working model of SUMO modification-mediated NR4A1 ubiquitination and degradation

NR4A1 by the E3 SUMO ligase PIAS3 allows the recruitment of the SUMO-interacting E3 ubiquitin ligase RNF4, which triggers the polyubiquitination and subsequent degradation of SUMOylated NR4A1. Thus, SENP1-mediated SUMO-deconjugation of NR4A1 greatly antagonizes its ubiquitination and subsequent proteosomal degradation.
Further, we demonstrated that a SUMO modificationdeficient NR4A1 mutant is more stable than the wild-type protein and has a much more potent transcriptional activity.

NR4A1 is an early response gene product; as such, the basal levels of NR4A1 are low and NR4A1 is degraded rapidly 
after induction. This latter property is often observed for early response gene products, as their activities are usually required only transiently to trigger a particular process. ${ }^{20}$ This is the first report of SUMOylation-triggered ubiquitinationmediated degradation of an early response gene product. Our findings, taken together with a recent proteomic study showing that at least $10 \%$ of SUMOylated proteins are ubiquitinated, ${ }^{51}$ suggest that SUMOylation-mediated ubiquitination may occur in other rapidly induced cellular responses.

NR4A1 stability and activity is known to be regulated by posttranslational modifications. Phosphorylation, which is mediated by various extracellular cues and protein kinases, determines the function of NR4A1 (refs 39-41). Acetylation and deacetylation induced by $\mathrm{P} 300$ and HDAC1 regulates NR4A1 protein turnover. ${ }^{37}$ We now report another type of regulation that is mediated by a different kind of reversible post-translational modification. It is not known whether NR4A1 phosphorylation modulates SUMOylation-triggered ubiquitination, and the known phosphorylation sites are not in very close proximity to the two SUMO attachment sites that we identified.

The two SUMOylation sites that we identified in NR4A1 are highly conserved and can be found in zebrafish, frogs and mammals. Inspection of the sequences of the NR4A1 paralogs, NR4A2 and NR4A3, reveals that these two isoforms also have these two SUMO conjugation sites. The consecutive SUMOylation and ubiquitination process is thus likely common to other members of the NR4A1 family of proteins.

NR4A1 associates with RNF4 in a SUMOylation-dependent manner, and SUMOylation-defective NR4A1 is more transcriptionally active. RNF4 interacts with transcription (co) factors. ${ }^{11,52-54}$ More recently, the interactome for RNF4 was determined using a proteomic approach, and many transcription factors were identified among its interaction partners. ${ }^{55}$ NR4A1 was not identified as an interaction partner, probably because it was expressed at low levels in those experimental conditions. Notably, RNF4 localizes to PML bodies. ${ }^{56}$ Interestingly, NR4A1 localizes to the nucleus when expressed alone, but when it is co-expressed with PML, both proteins co-localize in a speckled pattern in the nucleus (Supplementary FigureS1).

Future studies should investigate whether SUMOylationtriggered ubiquitination also plays a role in other NR4A1mediated effects downstream of other stimuli, such as tumourigenesis, glucose and lipid homeostasis, and vascular remodeling. Our results revealed the precise molecular mechanisms that regulate reversible SUMO modification of NR4A1 and control its stability. These mechanisms represent potential targets for therapeutic intervention in diseases linked to NR4A1 (dys) function.

\section{Materials and Methods}

Cell cultures. HEK293T and HeLa cells were cultured in Dulbecco's modified Eagle's medium. Jurkat, RAW264.7 and THP-1 cells were cultured in RPMI 1640 medium supplemented with 10\% FBS (Hyclone, Logan, Utah, USA) and $100 \mathrm{U} / \mathrm{ml}$ penicillin/streptomycin (Invitrogen, Carlsbad, CA, USA).

Plasmids and reagents. Flag-NR4A1 and Flag-NR4A1-2KR (K101/577R) were amplified from pCMV-SPORT6 NR4A1 (Open Biosystems, Huntsville, Alabama, USA) by PCR and inserted into the pLV-bc-CMV vector. The HA-SUMO1-3 and the SENP1 WT and CS constructs were described previously. ${ }^{57}$ HA-PIAS1, $-3, \mathrm{X} \alpha$ and $\mathrm{X} \beta$ were kindly provided by Dr. X.-H. Feng (Baylor College of Medicine), and Flag-PIAS3 was provided by Dr. D. Baker (Leiden
University Medical Center). V5-RNF4 and RNF4 shRNA ${ }^{58}$ were kindly provided by Dr. A. shRNAs targeting human PIAS3 (\#1, TRCN0000020784 and \#2, TRCN0000020786) were got from Sigma mission library. Vertegaal. 12-0tetradecanoylphorbol 13-acetate (TPA) and MG132 were purchased from Sigma (MO, USA).

We used the following antibodies: $\beta$-actin (A5441, Sigma, St Louis, MO,USA), c-Myc (a-14, sc-789, Santa Cruz Biotechnology, Santa Cruz, USA), HA (Y-11, sc-805, Santa Cruz Biotechnology), HA (12CA5, produced in our laboratory), Flag (M2, Sigma), ubiquitin (P4D1, Santa Cruz Biotechnology), V5 (Invitrogen). The anti-SUMO2/3 antibody was provided by Dr. A. Vertegaal.

Lentiviral transduction. Lentivirus was produced by co-transfecting $\mathrm{pLKO}-1$ (for the shRNA-knockdown) or pLV-bc-CMV (for cDNA expression) plasmids and the helper plasmids pCMV-VSVG, pMDLg-RRE (gag/pol), and pRSV-REV into HEK293T cells. Cell supernatants were collected $48 \mathrm{~h}$ after transfection and were either used promptly to infect cells or were stored at $-80^{\circ} \mathrm{C}$.

To obtain stable cell lines, cells were infected at low confluence $(20 \%)$ for $24 \mathrm{~h}$ with lentiviral supernatant that was diluted 1:1 with normal culture medium in the presence of $5 \mathrm{ng} / \mathrm{ml}$ polybrene (Sigma). Then, $48 \mathrm{~h}$ after infection, cells were placed under puromycin selection for 1 week and passaged before use.

Immunoprecipitation and immunoblotting. As described previously, ${ }^{59,60}$ cells were lysed by incubation with $1 \mathrm{ml}$ of lysis buffer $(20 \mathrm{~mm}$ Tris- $\mathrm{HCl}$, pH 7.4, 2 mM EDTA, 25 mm NaF, 1\% Triton X-100) plus protease inhibitors (Sigma) for $10 \mathrm{~min}$ at $4^{\circ} \mathrm{C}$. After centrifugation at $12000 \times \mathrm{g}$ for $15 \mathrm{~min}$, the lysates were immunoprecipitated with antibodies and protein A-Sepharose (GE Healthcare Bio-Sciences AB, Uppsala, Sweden) for $3 \mathrm{~h}$ at $4{ }^{\circ} \mathrm{C}$. Subsequently, the precipitates were washed three times with washing buffer $(50 \mathrm{~mm}$ Tris- $\mathrm{HCl}, \mathrm{pH} 8.0,150 \mathrm{~mm}$ $\mathrm{NaCl}, 1 \%$ Nonidet P- $40,0.5 \%$ sodium deoxycholate, and $0.1 \% \mathrm{SDS}$ ), and the immune complexes were eluted with sample buffer containing $1 \%$ SDS for 5 min at $95^{\circ} \mathrm{C}$ and analyzed by SDS-PAGE.

Immunoblotting was performed as described previously ${ }^{61}$ using secondary anti-mouse or anti-rabbit antibodies conjugated to horseradish peroxidase (GE Healthcare, Buckinghamshire, UK) plus visualization with chemiluminescence.

SUMOylation and ubiquitination assays. For the in vivo SUMOylation and ubiquitination assays, cells were washed with PBS (10 mM N-ethylmaleimide, NEM) and lysed in two pellet volumes of RIPA buffer (20 mM NaP (pH 7.4), $150 \mathrm{~mm}$ $\mathrm{NaCl}, 1 \%$ Triton, $0.5 \%$ sodium deoxycholate, and $1 \%$ SDS) supplemented with protease inhibitors and $10 \mathrm{~mm}$ NEM. Lysates were boiled, diluted with RIPA buffer containing $0.1 \%$ SDS, sonicated, and centrifuged at $4{ }^{\circ} \mathrm{C}(16,000 \times \mathrm{g})$ for $15 \mathrm{~min}$. The supernatant was incubated with specific antibodies plus sepharose for 2 to $6 \mathrm{~h}$ at $4{ }^{\circ} \mathrm{C}$. After extensive washing, bound proteins were eluted with $2 \times$ SDS sample buffer and separated on SDS-PAGE followed by immunoblotting.

Transcriptional reporter assay. Cells were transfected and lysed as described previously, 62,63 and luciferase activities were measured using a luminometer (Berthold Technologies, Bad Wildbad, Black Forest, Germany). Reporter activity was normalized to $\beta$-Gal activity from a cotransfected internal control plasmid. Experiments were performed in triplicate.

Statistical analysis. Statistical analyses were performed using a two-tailed unpaired $t$-test. $P<0.05$ was considered significant.

\section{Conflict of Interest}

The authors declare no conflict of interest.

Acknowledgements. We are grateful to Erik Meulmeester, Xin-hua Feng, Alfred Vertegaal Joost Schimmel, and David Baker for providing reagents and for valuable discussions. We thank Midory Thorikay and Maarten van Dinther for expert technical assistance. The current work was supported by special program from Ministry of Science and Technology of China (2016YFA0502500), the Chinese National Natural Science Funds (grant number 31571460, 31471315, K124924615, 81470851, R14C070002), the Jiangsu National Science Foundation BK20150354 and the Netherlands Organization for Health Research and Development (ZonMW grant no. 8238-20864), Netherlands Organization for Scientific Research (VICl 016.066.606) and by the Centre for Biomedical Genetics. 


\section{Author contributions}

$F Z, F X, L Z$ and JZ performed the experiments; FZ and LZ created the figures; and FZ, LZ and PtD wrote the paper.

1. Gareau JR, Lima CD. The SUMO pathway: emerging mechanisms that shape specificity, conjugation and recognition. Nat Rev Mol Cell Biol 2010; 11: 861-871.

2. Geiss-Friedlander R, Melchior F. Concepts in sumoylation: a decade on. Nat Rev Mol Cell Biol 2007; 8: 947-956.

3. Melchior F, Schergaut M, Pichler A. SUMO: ligases, isopeptidases and nuclear pores. Trends Biochem Sci 2003; 28: 612-618.

4. Johnson ES. Protein modification by SUMO. Annu Rev Biochem 2004; 73: 355-382.

5. Seeler JS, Dejean A. Nuclear and unclear functions of SUMO. Nat Rev Mol Cell Biol 2003; 4 : 690-699.

6. Hay RT. SUMO-specific proteases: a twist in the tail. Trends Cell Biol 2007; 17: 370-376.

7. Rodriguez MS, Dargemont C, Hay RT. SUMO-1 conjugation in vivo requires both a consensus modification motif and nuclear targeting. J Biol Chem 2001; 276: 12654-12659.

8. Tatham MH, Geoffroy MC, Shen L, Plechanovova A, Hattersley N, Jaffray EG et al. RNF4 is a poly-SUMO-specific E3 ubiquitin ligase required for arsenic-induced PML degradation. Nat Cell Biol 2008; 10: 538-546.

9. Matic I, Schimmel J, Hendriks IA, van Santen MA, van de Rijke F, van Dam H et al. Site-specific identification of SUMO-2 targets in cells reveals an inverted SUMOylation motif and a hydrophobic cluster SUMOylation motif. Mol Cell 2010; 39: 641-652.

10. Tatham MH, Jaffray E, Vaughan OA, Desterro JM, Botting $\mathrm{CH}$, Naismith JH et al. Polymeric chains of SUMO-2 and SUMO-3 are conjugated to protein substrates by SAE1/SAE2 and Ubc9. J Biol Chem 2001; 276: 35368-35374.

11. Abed M, Barry KC, Kenyagin D, Koltun B, Phippen TM, Delrow JJ et al. Degringolade, a SUMO-targeted ubiquitin ligase, inhibits Hairy/Groucho-mediated repression. EMBO J 2011 30: $1289-1301$.

12. Lallemand-Breitenbach V, Jeanne M, Benhenda S, Nasr R, Lei M, Peres $L$ et al. Arsenic degrades PML or PML-RARalpha through a SUMO-triggered RNF4/ubiquitin-mediated pathway. Nat Cell Biol 2008; 10: 547-555.

13. Sun H, Leverson JD, Hunter T. Conserved function of RNF4 family proteins in eukaryotes: targeting a ubiquitin ligase to SUMOylated proteins. EMBO J 2007; 26: 4102-4112.

14. Sun H, Hunter T. Poly-small ubiquitin-like modifier (PolySUMO)-binding proteins identified through a string search. J Biol Chem 2012; 287: 42071-42083.

15. Poulsen SL, Hansen RK, Wagner SA, van Cuijk L, van Belle GJ, Streicher W et al. RNF111/ Arkadia is a SUMO-targeted ubiquitin ligase that facilitates the DNA damage response. J Cell Biol 2013; 201: 797-807.

16. van Cuijk L, van Belle GJ, Turkyilmaz Y, Poulsen SL, Janssens RC, Theil AF et al. SUMO and ubiquitin-dependent XPC exchange drives nucleotide excision repair. Nat Commun 2015; 6: 7499 .

17. Wang Z, Benoit G, Liu J, Prasad S, Aarnisalo P, Liu X et al. Structure and function of Nurr1 identifies a class of ligand-independent nuclear receptors. Nature 2003; 423: 555-560.

18. Flaig R, Greschik H, Peluso-lltis C, Moras D. Structural basis for the cell-specific activities of the NGFI-B and the Nurr1 ligand-binding domain. J Biol Chem 2005; 280: 19250-19258.

19. Mohan HM, Aherne CM, Rogers AC, Baird AW, Winter DC, Murphy EP. Molecular pathways: the role of NR4A orphan nuclear receptors in cancer. Clin Cancer Res 2012; 18: 3223-3228.

20. Zhao Y, Bruemmer D. NR4A orphan nuclear receptors: transcriptional regulators of gene expression in metabolism and vascular biology. Arterioscler Thromb Vasc Biol 2010; 30: 1535-1541.

21. Pearen MA, Muscat GE. Minireview: nuclear hormone receptor $4 \mathrm{~A}$ signaling: implications for metabolic disease. Mol Endocrinol 2010; 24: 1891-1903.

22. Zhou F, Drabsch Y, Dekker TJ, de Vinuesa AG, Li Y, Hawinkels LJ et al. Nuclear receptor NR4A1 promotes breast cancer invasion and metastasis by activating TGF-beta signalling. Nat Commun 2014; 5: 3388.

23. Hazel TG, Nathans D, Lau LF. A gene inducible by serum growth factors encodes a member of the steroid and thyroid hormone receptor superfamily. Proc Natl Acad Sci USA 1988; 85: 8444-8448.

24. Williams GT, Lau LF. Activation of the inducible orphan receptor gene nur77 by serum growth factors: dissociation of immediate-early and delayed-early responses. $\mathrm{Mol}$ Cell Biol 1993; 13: 6124-6136.

25. Martinez-Gonzalez J, Badimon L. The NR4A subfamily of nuclear receptors: new early genes regulated by growth factors in vascular cells. Cardiovasc Res 2005; 65: 609-618.

26. Woronicz JD, Calnan B, Ngo V, Winoto A. Requirement for the orphan steroid receptor Nur77 in apoptosis of T-cell hybridomas. Nature 1994; 367: 277-281.

27. Mullican SE, Zhang S, Konopleva M, Ruvolo V, Andreeff M, Milbrandt J et al. Abrogation of nuclear receptors $\mathrm{Nr} 4 \mathrm{a} 3$ and $\mathrm{Nr} 4 \mathrm{a} 1$ leads to development of acute myeloid leukemia. Nat Med 2007; 13: 730-735.

28. Ramirez-Herrick AM, Mullican SE, Sheehan AM, Conneely OM. Reduced NR4A gene dosage leads to mixed myelodysplastic/myeloproliferative neoplasms in mice. Blood 2011; 117: 2681-2690.

29. Li H, Kolluri SK, Gu J, Dawson MI, Cao X, Hobbs PD et al. Cytochrome c release and apoptosis induced by mitochondrial targeting of nuclear orphan receptor TR3. Science 2000; 289: $1159-1164$.
30. Kim SO, Ono K, Tobias PS, Han J. Orphan nuclear receptor Nur77 is involved in caspaseindependent macrophage cell death. J Exp Med 2003; 197: 1441-1452.

31. van Tiel CM, Kurakula K, Koenis DS, van der Wal E, de Vries CJ. Dual function of Pin1 in NR4A nuclear receptor activation: enhanced activity of NR4As and increased Nur77 protein stability. Biochim Biophys Acta 2012; 1823: 1894-1904.

32. Chen HZ, Li L, Wang WJ, Du XD, Wen Q, He JP et al. Prolyl isomerase Pin1 stabilizes and activates orphan nuclear receptor TR3 to promote mitogenesis. Oncogene 2012; 31: 2876-2887.

33. Arkenbout EK, de Waard V, van Bragt M, van Achterberg TA, Grimbergen JM, Pichon B et al. Protective function of transcription factor TR3 orphan receptor in atherogenesis: decreased lesion formation in carotid artery ligation model in TR3 transgenic mice. Circulation 2002; 106: 1530-1535.

34. You B, Jiang YY, Chen S, Yan G, Sun J. The orphan nuclear receptor Nur77 suppresses endothelial cell activation through induction of IkappaBalpha expression. Circ Res 2009; 104: 742-749.

35. Pei L, Waki H, Vaitheesvaran B, Wilpitz DC, Kurland IJ, Tontonoz P. NR4A orphan nuclear receptors are transcriptional regulators of hepatic glucose metabolism. Nat Med 2006; 12: 1048-1055.

36. Park KC, Song KH, Chung HK, Kim H, Kim DW, Song JH et al. CR6-interacting factor 1 interacts with orphan nuclear receptor Nur77 and inhibits its transactivation. Mol Endocrinol 2005; 19: 12-24.

37. Kang SA, Na H, Kang HJ, Kim SH, Lee MH, Lee MO. Regulation of Nur77 protein turnover through acetylation and deacetylation induced by p300 and HDAC1. Biochem Pharmacol 2010; 80: 867-873.

38. Wansa KD, Muscat GE. TRAP220 is modulated by the antineoplastic agent 6-Mercaptopurine, and mediates the activation of the NR4A subgroup of nuclear receptors. $\mathrm{J} \mathrm{Mol} \mathrm{Endocrinol} \mathrm{2005;} \mathrm{34:} \mathrm{835-848.}$

39. Davis IJ, Hazel TG, Chen RH, Blenis J, Lau LF. Functional domains and phosphorylation of the orphan receptor Nur77. Mol Endocrinol 1993; 7: 953-964.

40. Pekarsky Y, Hallas C, Palamarchuk A, Koval A, Bullrich F, Hirata Y et al. Akt phosphorylates and regulates the orphan nuclear receptor Nur77. Proc Natl Acad Sci USA 2001; 98: 3690-3694.

41. Liu B, Wu JF, Zhan YY, Chen HZ, Zhang XY, Wu Q. Regulation of the orphan receptor TR3 nuclear functions by c-Jun $\mathrm{N}$ terminal kinase phosphorylation. Endocrinology 2007; 148 : 34-44.

42. Katagiri $Y$, Hirata $Y$, Milbrandt J, Guroff G. Differential regulation of the transcriptional activity of the orphan nuclear receptor NGFI-B by membrane depolarization and nerve growth factor. J Biol Chem 1997; 272: 31278-31284.

43. Wingate AD, Campbell DG, Peggie M, Arthur JS. Nur77 is phosphorylated in cells by RSK in response to mitogenic stimulation. Biochem J 2006; 393: 715-724.

44. Yao LM, He JP, Chen HZ, Wang Y, Wang WJ, Wu R et al. Orphan receptor TR3 participates in cisplatin-induced apoptosis via Chk2 phosphorylation to repress intestinal tumorigenesis. Carcinogenesis 2012; 33: 301-311.

45. Geoffroy MC, Jaffray EG, Walker KJ, Hay RT. Arsenic-induced SUMO-dependent recruitment of RNF4 into PML nuclear bodies. Mol Biol Cell 2010; 21: 4227-4239.

46. Geoffroy MC, Hay RT. An additional role for SUMO in ubiquitin-mediated proteolysis. Nat Rev Mol Cell Biol 2009; 10: 564-568.

47. Rojas-Fernandez A, Plechanovova A, Hattersley N, Jaffray E, Tatham MH, Hay RT. SUMO chain-induced dimerization activates RNF4. Mol Cell 2014; 53: 880-892.

48. Winoto A, Littman DR. Nuclear hormone receptors in T lymphocytes. Cell 2002; 109(Suppl): S57-S66.

49. Beg AA, Baltimore D. An essential role for NF-kappaB in preventing TNF-alpha-induced cell death. Science 1996; 274: 782-784.

50. Van Antwerp DJ, Martin SJ, Kafri T, Green DR, Verma IM. Suppression of TNF-alphainduced apoptosis by NF-kappaB. Science 1996; 274: 787-789.

51. Matic I, van Hagen M, Schimmel J, Macek B, Ogg SC, Tatham MH et al. In vivo identification of human small ubiquitin-like modifier polymerization sites by high accuracy mass spectrometry and an in vitro to in vivo strategy. Mol Cell Proteomics 2008; 7: 132-144.

52. Moilanen AM, Poukka H, Karvonen U, Hakli M, Janne OA, Palvimo JJ. Identification of a novel RING finger protein as a coregulator in steroid receptor-mediated gene transcription. Mol Cell Biol 1998; 18: 5128-5139.

53. Kaiser FJ, Moroy T, Chang GT, Horsthemke B, Ludecke HJ. The RING finger protein RNF4, a co-regulator of transcription, interacts with the TRPS1 transcription factor. J Biol Chem 2003; 278: 38780-38785

54. Wu SM, Kuo WC, Hwu WL, Hwa KY, Mantovani R, Lee YM. RNF4 is a coactivator for nuclear factor $Y$ on GTP cyclohydrolase I proximal promoter. Mol Pharmacol 2004; 66: 1317-1324.

55. Bruderer R, Tatham MH, Plechanovova A, Matic I, Garg AK, Hay RT. Purification and identification of endogenous polySUMO conjugates. EMBO Rep 2011; 12: 142-148.

56. Percherancier Y, Germain-Desprez D, Galisson F, Mascle XH, Dianoux L, Estephan P et al. Role of SUMO in RNF4-mediated promyelocytic leukemia protein (PML) degradation: sumoylation of PML and phospho-switch control of its SUMO binding domain dissected in living cells. J Biol Chem 2009; 284: 16595-16608.

57. Meulmeester E, Kunze M, Hsiao HH, Urlaub H, Melchior F. Mechanism and consequences for paralog-specific sumoylation of ubiquitin-specific protease 25. Mol Cell 2008; 30: 610-619. 
58. van Hagen M, Overmeer RM, Abolvardi SS, Vertegaal AC. RNF4 and VHL regulate the proteasomal degradation of SUMO-conjugated hypoxia-inducible factor-2alpha. Nucleic Acids Res 2010; 38: 1922-1931.

59. Zhang L, Zhou F, Drabsch Y, Gao R, Snaar-Jagalska BE, Mickanin C et al. USP4 is regulated by AKT phosphorylation and directly deubiquitylates TGF-beta type I receptor. Nat Cell Biol 2012; 14: 717-726.

60. Zhang L, Huang H, Zhou F, Schimmel J, Pardo CG, Zhang T et al. RNF12 controls embryonic stem cell fate and morphogenesis in zebrafish embryos by targeting Smad7 for degradation. Mol Cell 2012; 46: 650-661.
61. Zhou F, Zhang L, Wang A, Song B, Gong K, Hu M et al. The association of GSK3 beta with E2F1 facilitates nerve growth factor-induced neural cell differentiation. J Biol Chem 2008; 283: 14506-14515.

62. Zhang Y, Liu S, Mickanin C, Feng Y, Charlat O, Michaud GA et al. RNF146 is a poly(ADPribose)-directed E3 ligase that regulates axin degradation and Wnt signalling. Nat Cell Biol 2011; 13: 623-629.

63. Zhou $F$, Zhang L, van Laar T, van Dam H, Ten Dijke P. GSK3beta inactivation induces apoptosis of leukemia cells by repressing the function of c-Myb. Mol Biol Cell 2011; 22 3533-3540.

Supplementary Information accompanies this paper on Cell Death and Differentiation website (http://www.nature.com/cdd) 